\title{
Neurotrophin-3 Expressed In Situ Induces Axonal Plasticity in the Adult Injured Spinal Cord
}

\author{
Lijun Zhou, ${ }^{1,2}$ Brian J. Baumgartner, ${ }^{1}$ Sandra J. Hill-Felberg, ${ }^{1}$ Leonard R. McGowen, ${ }^{1,2}$ and H. David Shine ${ }^{1,2,3,4}$ \\ ${ }^{1}$ Department of Neurosurgery, ${ }^{2}$ Center for Cell and Gene Therapy, ${ }^{3}$ Division of Neuroscience, and ${ }^{4}$ Department of Molecular and Cellular Biology, Baylor \\ College of Medicine, Houston, Texas 77030
}

\begin{abstract}
The mammalian CNS lacks the ability to effectively compensate for injury by the regeneration of damaged axons or axonal plasticity of intact axons. However, reports suggest that molecular or cellular manipulations can induce compensatory processes that could support regeneration or plasticity after trauma. We tested whether local, sustained release of the neurotrophic factor neurotrophin-3 (NT-3) would support axonal plasticity in the spinal cord distal to the site of injury in rats. The corticospinal tract (CST) was cut unilaterally at the level of the medulla. This avoided excessive inflammation, secondary cell death, vascular disruption, and the release of inhibitory molecules in the lumbar spinal cord. A replication-defective adenoviral vector (Adv) carrying the NT-3 gene (Adv.EF $\alpha$-NT3) was delivered to the spinal motoneurons by retrograde transport through the sciatic nerve. Retrograde transport of the adenoviral vectors avoided the inflammatory response that would be associated with direct injection into the spinal cord. Transduction of spinal motoneurons with Adv.EF $\alpha$-NT3 resulted in a significant increase in the concentration of NT-3 in the L3-L6 region of the spinal cord for up to 3 weeks. In animals with a CST lesion, this local expression of NT-3 induced growth of axons from the intact CST across the midline to the denervated side. If the CST remained intact, overexpression of NT-3 did not lead to an increase in the number of axons crossing the midline. These data demonstrate that local, sustained expression of NT-3 will support axonal plasticity of intact CST axons after trauma-induced denervation.
\end{abstract}

Key words: neurotrophin-3; axonal plasticity; adenoviral vectors; spinal cord injury; regeneration; NT-3

\section{Introduction}

The robust anatomical and functional plasticity of the embryonic and perinatal mammalian CNS is lost soon after birth, so that regeneration or compensation after injury is all but absent in adults. The lack of a supportive environment and the presence of inhibitory molecules prevent the regeneration of damaged axons and compensatory growth of intact axons. This inability of the adult CNS to regenerate or effectively compensate after injury leads to permanent and many times to devastating functional deficiencies after injury. Nevertheless, there is emerging evidence that the adult CNS retains some capacity for plasticity and may, under the right conditions, be able to regenerate (Schwab, 2002). Manipulation of the CNS environment will support sprouting of undamaged axons into areas denervated by lesions. Neutralization of the myelin-associated neurite growth inhibitor Nogo with a monoclonal antibody (IN-1) resulted in extensive sprouting of axons from an intact corticospinal tract (CST) to the denervated side of the spinal cord after the other tract was cut at the level of the pyramids (Thallmair et al., 1998). In the same CST lesion

\footnotetext{
Received Sept. 16, 2002; revised Nov. 20, 2002; accepted Nov. 21, 2002.

This work was supported by National Institutes of Health Grant NS35280 and by Mission Connect, a project of The Institute for Rehabilitation and Research Foundation. We thank our colleagues of Mission Connect helpful discussion of this work; D. Bui, T. Chacko, and R. Rosado for technical assistance; R. J. Gill for immunocytochemical analysis; S. L. C. Woo and Z. S. Guo for the pXCJL.1 plasmid; and John Gilbert for editorial assistance.

Correspondence should be addressed to Dr. H. David Shine, Department of Neurosurgery, Baylor College of Medicine, One Baylor Plaza, Houston, TX 77030. E-mail: hshine@bcm.tmc.edu.

B. J. Baumgartner's present address: Department of Biology, Trinity Valley Community College, Athens, TX 75751.

S. J. Hill-Felberg's present address: Department of Medicine, Tulane University Health Sciences Center, New Orleans, LA 70112.

Copyright $\odot 2003$ Society for Neuroscience $\quad$ 0270-6474/03/231424-08\$15.00/0
}

(CSTL) model, prevention of myelination by $\mathrm{x}$-irradiation in rats reduced the amount of myelin-associated inhibitors, permitting axons to sprout from the intact CST into the denervated side (Vanek et al., 1998). Sustained cortical delivery of inosine stimulated extensive collateral sprouting of rat CST after injury by an undefined intracellular mechanism (Benowitz et al., 1999). Locally injected neurotrophin-3 (NT-3) enhances the regenerative sprouting of transected CST (Schnell et al., 1994). These experiments indicated that providing enough promoting factors or blocking inhibitory molecules allows intact axon growth and plasticity in the adult spinal cord. Together, the observations that molecular and cellular manipulations of the CNS will induce regeneration and compensation suggest that the adult CNS has not completely lost its ability to respond to trauma.

We have demonstrated previously that the retrograde transport of adenoviral vectors (Advs) expressing neurotrophic factor genes (Adv.RSV-nf) from the periphery to CNS motoneurons results in the local expression of neurotrophic factors that block axotomy-induced motoneuron death in the facial nucleus and spinal cord (Baumgartner and Shine, 1997, 1998a) and maintain motor function (Baumgartner and Shine, 1998b). These experiments demonstrated that biologically active neurotrophic factors could be locally expressed in the CNS without vector-associated trauma and inflammation and in sufficient quantities and duration to have neuroprotective effects. In the present study, we used retrograde delivery of an adenoviral vector carrying the gene for NT-3 to express this neurotrophic factor locally in motoneurons in the spinal cord; we also tested whether this local expression would induce and support the growth of axons from the spared CST in the unilateral CSTL model. 


\section{Materials and Methods}

Preparation of an adenoviral vector carrying the NT-3 gene. To express NT-3 locally in the rat spinal cord, we constructed a replication-defective Adv carrying the cDNA sequence of rat NT-3, the nerve growth factor (NGF) signal sequence, and the FLAG marker sequence driven by the mammalian elongation factor $\alpha(\mathrm{EF} \alpha)$ promoter (Kim et al., 1990, 1993). The rat cDNA and signal peptide sequences were obtained from the plasmid pADL.1/RSV-NT-3 used by us previously (Baumgartner and Shine, 1998a) and fitted with the FLAG marker sequence (Hopp et al., 1988 ) at the $3^{\prime}$ end. This cassette was ligated into the multiple cloning site of pXCJL.1 (provided by S. L. C. Woo and Z. S. Guo, Baylor College of Medicine, Houston, TX), which contains the human EF $\alpha$ promoter sequence at the $5^{\prime}$ end and the bovine growth hormone gene polyadenylation signal sequence at the $3^{\prime}$ end (Guo et al., 1996). Others have demonstrated that the mammalian $\mathrm{EF} \alpha$ promoter is optimal for high, long-term gene expression in vivo (Guo et al., 1996). The methods of Graham and Prevec (1991) were used to construct replication-defective recombinant Adv via homologous recombination with the plasmid pJM17 (which contains the E1A-deleted Adv type 5 genome). The recombinant pXCJL.1 plasmid containing an NT-3 expression cassette was cotransfected with pJM17 into human embryonic kidney 293 cells, and plaques were allowed to develop over 2 weeks. PCR with primers specific for the NT-3 construct was used to confirm that the resultant Adv.EF $\alpha$ NT3 vector contained the correct sequence. The resulting Adv.EF $\alpha$-NT3 plaques were purified by two rounds of "plaque purification," and largescale production and $\mathrm{CsCl}$ purification of Adv.EF $\alpha$-NT3 were performed as described by Graham and Prevec (1991). The viral titer was determined by plaque assay. An adenoviral vector carrying the LacZ gene (Adv.EF $\alpha$-LacZ) was used as a control.

Western blot and ELISA analyses of NT-3-FLAG expressed by HeLa cells transduced with $A d v . E F \alpha-N T 3$ in vitro. Adv.EF $\alpha$-NT3 or Adv.EF $\alpha-L a c Z$ was added at a multiplicity of infection (MOI) of 100 to cultures of HeLa cells grown in DMEM (Invitrogen, Carlsbad, CA) supplemented with $10 \%$ fetal bovine serum (FBS) when the cells were $80 \%$ confluent. After $12 \mathrm{hr}$, the medium was changed to serum-free Opti-MEM (Invitrogen). After $48 \mathrm{hr}$, the conditioned media were collected, concentrated with molecular weight (MW) 3000 cutoff SpinX-UF3 centrifuge filters (Costar, Cambridge, MA), and analyzed by Western blotting for the presence of the FLAG marker peptide using standard techniques used by us previously to characterize other vectors (Baumgartner and Shine, 1997). Proteins containing the FLAG peptide were detected with an antiFLAG monoclonal antibody (M2; Sigma, St. Louis, MO) at a 1:1000 dilution and the anti-mouse Vectastain ABC kit (Vector Laboratories, Burlingame, CA). Bovine alkaline phosphatase (BAP) labeled with the FLAG peptide (BAP-FLAG; Sigma) was used as a positive control. The quantity of NT-3 protein in the conditioned media was determined by ELISA (Promega, Madison, WI).

Analysis of biological activity of conditioned medium from HeLa cells transduced with $A d v$.EF $\alpha-N T 3$ on chick sensory neurons. HeLa cells grown in DMEM supplemented with 10\% FBS were transduced with Adv.EF $\alpha$ NT3 or Adv.EF $\alpha-L a c Z$ at MOIs of 100 and cultured for $12 \mathrm{hr}$. The culture medium was replaced with fresh serum-free Opti-MEM medium (Invitrogen), and after $48 \mathrm{hr}$ the conditioned medium was collected and analyzed for NT-3 activity in sensory neuron cultures. Primary cultures (Davies, 1989) of dissociated chick embryo dorsal root ganglia (DRGs) at embryonic day 10 were grown in DMEM with $10 \%$ FBS supplemented with $100 \mathrm{ng} / \mathrm{ml}$ recombinant NT-3 (Promega) or 25\% v/v conditioned media from HeLa cell cultures transduced with Adv.EF $\alpha$-NT3 or Adv.EF $\alpha$-LacZ mixed with DMEM and 10\% FBS. Each treatment was done in triplicate. After $2 \mathrm{~d}$ in culture, the number of surviving neurons was counted at $100 \times$ magnification. Neurons with bright bodies and neurites extending more than three times the cell body diameter were counted as surviving neurons. Ten fields per culture well were counted and summed for each well.

Pyramidotomy for unilateral lesion of the CST. In the rat, most CST fibers decussate at the level of the pyramids in the brainstem and project to the contralateral side of the spinal cord via the dorsal funiculus. The CST was cut at the level of the pyramids above the decussation, resulting

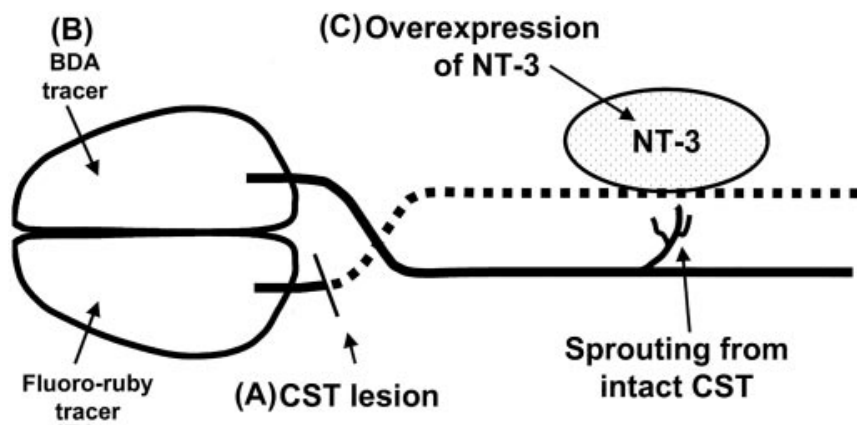

(B)

Figure 1. Schematic of the experimental protocol. $A, 0$ ne CST was lesioned at the level of the hindbrain. $B$, After $10 \mathrm{~d}$, the neuronal tracers BDA and Fluoro-ruby were injected into opposite sensorimotor cortices. C, Four days after the tracer injection, Adv.EF $\alpha$-NT3 or Adv.EF $\alpha$-LacZ was delivered by retrograde transport to the spinal motoneurons in which NT-3 or LacZ were overexpressed.

in an almost complete denervation of the right CST in the spinal cord (Fig. 1). Severing the CST unilaterally at the pyramids permitted us to denervate the CST to the full extent of the length of the spinal cord, thus avoiding vasculature disruption and inflammation that would be associated with a lesion in the spinal cord. Adult Sprague Dawley female rats (250-300 gm) were anesthetized with halothane followed by continuous isoflurane using a vaporizing system (Vip 3000; Matrx Medical Inc., Orchard Park, NY). A midline incision was made in the ventral neck region. The trachea and paratracheal musculature were retracted to expose the basioccipital portion of the skull. The left CST was exposed by a $2 \mathrm{~mm}$ craniotomy performed with a burr drill just lateral to the midline ridge. Taking the basal artery as the landmark of midline, a 1.5-mm-wide and $0.5-\mathrm{mm}$-deep incision was made into the medulla. To ensure that all of the CST was completely transected, we aspirated the incision site with a fine-tipped glass suction pipette. The exposed brain tissue was covered with gel foam, and the skin was closed with sutures. Animal experiments and animal care were performed in accordance with approved protocols of the Baylor College of Medicine.

Anterograde tracing of CST projections. Ten days after CSTL, the rats were anesthetized with isoflurane and fixed in a sterotaxic frame. Biotinylated dextran amine (BDA; lysine fixable, MW 10,000; Molecular Probes, Eugene, OR) at a concentration of $10 \%$ in PBS was injected into 12 sites ( $146 \mathrm{nl}$ per site) in two rows of six sites spaced $0.5 \mathrm{~mm}$ apart caudorostrally and $1 \mathrm{~mm}$ apart mediolaterally at a depth of $1.2 \mathrm{~mm}$ in the sensorimotor cortex on the side that innervated the intact CST (Grill et al., 1997) using a Nanoliter Injector (World Precision Instruments, Sarasota, FL) fitted with a glass pipette with a $40 \mu \mathrm{m}$ tip. Tetramethylrhodamine dextran (Fluoro-ruby, 10\% in PBS; Molecular Probes) was injected in the same manner as the BDA into the other side of the cortex to examine the completeness of the CSTL. Rats found to have Fluoro-rubypositive axons in the lesioned CST were excluded from analysis, because their presence indicated that the lesion was incomplete.

Retrograde delivery of adenoviral vectors. Two weeks after CSTL and $4 \mathrm{~d}$ after BDA cortical injection, the rats were anesthetized with continuous isoflurane, and an incision was made posterior and parallel to the femur on the right side to expose the sciatic nerve within its surrounding connective tissue and superficial blood vessels. The sciatic nerve was transected $\sim 2 \mathrm{~mm}$ proximal to the bifurcation of the common peroneal and tibial branches. The proximal end of the cut nerve was placed in a small chamber fashioned from a $1.5 \mathrm{~cm}$ length of polyethylene tubing (1.4 mm inner diameter; Intramedic; Becton Dickinson, Sparks, MD) with one end sealed with a 2-mm-diameter glass bead and filled with $1 \times$ $10^{9}$ infectious units of either Adv.EF $\alpha$-NT3 or Adv.EF $\alpha$-LacZ. After the nerve stump was inserted into the chamber, $\sim 2 \mu$ l of sterile Cello-Seal (Fisher Scientific, Fairlawn, NJ) was injected into the opening of the tube around the nerve using a $1 \mathrm{ml}$ syringe with a 27 gauge needle (Becton Dickinson, Franklin Lake, NJ) to prevent the adenoviral solution from leaking out. The chamber was fixed in place with cyanoacrylate glue $(3 \mathrm{M}$, St. Paul, MN), and the muscles and skin were sutured closed. 
Adenoviral vector-mediated expression of NT-3 in vivo. Adv.EF $\alpha$-NT3, Adv.EF $\alpha-L a c Z$, PBS, or nothing (sham surgery) was delivered to the spinal motoneurons by retrograde transport as described above to four animals in each treatment group. Three weeks later the animals were killed under anesthesia, and the fresh lumbar spinal cords from L3 to L6 were dissected out, cut longitudinally along the dorsal medial sulcus into two parts, and frozen immediately at $-80^{\circ} \mathrm{C}$. The frozen tissue was homogenized in a lysate buffer consisting of $137 \mathrm{~mm} \mathrm{NaCl}, 20 \mathrm{~mm}$ Tris- $\mathrm{HCl}$, pH 7.6, 1\% Igepal CA-630, 10\% glycerol, 1 mM PMSF, $10 \mu \mathrm{g} / \mathrm{ml}$ aprotinin, $1 \mu \mathrm{g} / \mathrm{ml}$ leupeptin, and $0.5 \mathrm{~mm}$ sodium vanadate (all reagents from Sigma) and centrifuged at $13,000 \times g$ at $4^{\circ} \mathrm{C}$ for $30 \mathrm{~min}$ to remove the nonsoluble fraction. The concentration of NT-3 in the supernatant was determined by ELISA (Promega).

Histochemistry. Three weeks after vector delivery, the animals were heavily anesthetized with halothane and perfused intracardially with heparinized PBS followed by $4 \%$ paraformaldehyde (PFA) in PBS. The lumbar region of the spinal cords (L3-L6) was removed and postfixed in the same buffered fixative for $3 \mathrm{hr}$, washed briefly in PBS, and infiltrated with $21 \%$ sucrose in PBS. Cross sections of spinal cord $40 \mu \mathrm{m}$ thick were cut on a cryostat and collected in PBS. Every sixth section was put in one well of a 96 well culture plate. One section per two wells was picked randomly for BDA staining. A total of 14 sections between lumbar segments L3 and L6 were analyzed for each animal. For BDA staining, sections were washed in PBS three times, for $15 \mathrm{~min}$ each, followed by treatment with $0.3 \% \mathrm{H}_{2} \mathrm{O}_{2}$ for $30 \mathrm{~min}$ at room temperature. After three washes in PBS containing $0.1 \%$ Triton X-100 (PBST), BDA was detected with $\mathrm{ABC}$ reagent (Vector Laboratories) and diaminobenzidine (DAB). CST axons containing the anterograde marker BDA developed a darkbrown reaction product that was readily visible against the unstained spinal cord tissue.

To examine the completeness of the CSTL, BDA was detected with Alexa Fluor 488-conjugated streptavidin (Molecular Probes). Lumbar spinal cord sections were washed in PBST, incubated with Alexa Fluor 488 -conjugated streptavidin $(1 \mu \mathrm{g} / \mathrm{ml}$ in PBST) overnight, washed in PBS, and mounted with SuperMount (BioGenex, San Ramon, CA). Standard immunocytochemical techniques were used to detect macrophages in the lumbar spinal cord using ED1 antibody (Serotec, Oxford, UK) and a goat anti-mouse secondary antibody linked to Alexa Fluor 568 dye (Molecular Probes). To demonstrate the expression of LacZ in motoneurons after the retrograde delivery of Adv.EF $\alpha-L a c Z$, spinal cord sections were stained for $\beta$-galactosidase ( $\beta$-gal) activity using a standard histochemical method. One week after the retrograde delivery of Adv.EF $\alpha$ $L a c Z$, the rats were perfusion-fixed with $4 \%$ PFA and $40 \mu \mathrm{m}$ cross sections were taken from the lumbar spinal cord. After three washes in PBS, the sections were incubated for $30 \mathrm{~min}$ at $37^{\circ} \mathrm{C}$ in a solution of $0.5 \mathrm{mg} / \mathrm{ml}$ 5 -bromo-4-chloro-3-indolyl- $\beta$-D-galactopyranoside (X-gal; Sigma) in $44 \mathrm{~mm}$ HEPES buffer, $\mathrm{pH} 7.4$, containing (in $\mathrm{mm}$ ): $3 \mathrm{~K}_{3} \mathrm{Fe}(\mathrm{CN})_{6}, 3$ $\mathrm{K}_{4} \mathrm{Fe}(\mathrm{CN})_{6}, 15 \mathrm{NaCl}$, and $1.3 \mathrm{MgCl}_{2}$. Washing the sections in PBS stopped the reaction.

To determine the survival of motoneurons after the retrograde delivery of adenoviral vectors, five sections from three rats from each group were stained with hematoxylin and eosin. Large, multipolar cells located in the ventral horn of the spinal cord were identified as motoneurons and counted. The number of motoneurons on the lesioned and unlesioned sides of the spinal cords were compared within each group using a paired $t$ test. The ratio of the number of motoneurons on the lesioned side to the number of motoneurons on the unlesioned side was compared; this compensated for variability between animals.

Quantification of CST axons. Photomicrographs of the spinal cord sections were taken with a digital camera (AxioCam; Carl Zeiss, Jena, Germany) at $100 \times$ magnification under dark-field illumination. Five vertical lines, $M, N_{1}, N_{2}, L_{1}$, and $L_{2}$, were drawn on the photographs (see Fig. $7 a$ ), where $M$ passed through the midline, $N_{1}$ was just lateral to the funiculus of the normal side of the CST parallel to $M$, and $N_{2}$ was parallel to $N_{1}$ and twice the distance of $N_{1}$ to $M$. On the lesioned side of the spinal cord, $L_{1}$ and $L_{2}$ corresponded to lines of $N_{1}$ and $N_{2}$. Lines $A$ and $B$ were drawn perpendicular to the dorsoventral axis of the cord. Line $A$ was drawn just below the dorsal columns, and line $B$ was drawn just above the ventral columns. Axons that crossed the five lines between lines $A$ and $B$ were counted on the computer screen. To compensate for any variability in the degree of BDA staining, we calculated the ratios of $M, L_{1}$ and $L_{2}$ to $N_{1}+N_{2}\left(M / N_{1}+N_{2}, L_{1} / N_{1}+N_{2}, L_{2} / N_{1}+N_{2}\right)$.

Statistical analysis. ANOVA followed by the appropriate parametric and nonparametric post hoc tests were used for multiple group comparisons and Student's $t$ test for paired comparisons using SigmaStat software (SPSS Inc., Chicago, IL). Significance was assigned at $p<0.05$.

\section{Results}

HeLa cells transduced in vitro with Adv.EF $\alpha$-NT3 express and secrete biologically active NT-3

In previous reports we used the Rous sarcoma virus (RSV) promoter to control the expression of neurotrophic factors in $\mathrm{Adv}$ constructs (Adv.RSV-nf) (Baumgartner and Shine, 1997, $1998 \mathrm{a}, \mathrm{b})$. To verify that the $\mathrm{EF} \alpha$ promoter efficiently drove the expression of the NT-3 gene in the Adv.EF $\alpha$-NT3 construct, we repeated the in vitro analyses that were performed to characterize Adv.RSV- $n f$ constructs (Baumgartner and Shine, 1997). Western blot analysis of the conditioned culture medium from HeLa cells transduced with Adv.EF $\alpha$-NT3 at an MOI of 100 revealed a strong FLAG-positive band with the predicted size for the recombinant NT-3-FLAG hybrid protein (Fig. 2a). By ELISA analysis of the conditioned medium, the mean \pm SD synthesis rate of NT-3 was $0.76 \pm 0.35 \mu \mathrm{g}$ per $10^{6}$ cells per day. The biological activity of the NT-3 produced by HeLa cells transduced with Adv.EF $\alpha$-NT3 was measured by culturing embryonic sensory neurons from dissociated DRG in conditioned medium. The results were compared with the activities of conditioned medium from cells transduced with Adv.EF $\alpha-L a c Z$, with conditioned medium from untransduced HeLa cells, and with commercial NT-3 at $100 \mathrm{ng} /$ $\mathrm{ml}$. The conditioned medium from HeLa cells that had been transduced with the Adv.EF $\alpha$-NT3 had a significantly greater survival effect on DRG neurons (Fig. $2 b$ ) than did medium removed from cells transduced with Adv.EF $\alpha-L a c Z(p<0.01$; ANOVA followed by Student-Newman-Keuls test; $\mathrm{df}=3 ; F=$ 13.274).

\section{Transgene expression after retrograde transport of adenoviral vectors from sciatic nerve to the spinal cord}

Adv.EF $\alpha$-NT3 and Adv.EF $\alpha$-LacZ were delivered to the spinal motoneurons through the sciatic nerve by retrograde transport. One week later, histochemical staining for the presence of $\beta$-gal in the lumbar spinal cord showed that many motoneurons expressed the LacZ gene (Fig. 3). The X-gal reaction product was present not only in the motoneuron cell bodies but also in many of their dendrites that projected to the midline region of the spinal cord.

ELISA analysis (Fig. 4) of the concentration of NT-3 in the lumbar spinal cord on the side that received vector showed that the animals receiving Adv.EF $\alpha$-NT3 had significantly greater amounts of NT-3 than those receiving Adv.EF $\alpha$-LacZ, PBS, or sham surgery in both lesioned animals $(p<0.05$; ANOVA followed by the Student-Newman-Keuls test; df $=3 ; H=9.265$ ) and nonlesioned animals $(p<0.05$; ANOVA followed by the Student-Newman-Keuls test; $\mathrm{df}=3 ; F=11.696$ ). There was no significant difference between the Adv.EF $\alpha$-NT3 group with CSTL and the Adv.EF $\alpha$-NT3 group without lesions ( $p=0.938$; Student's $t$ test; $\mathrm{df}=6 ; t=0.0814)$. At 3 weeks after the delivery of Adv.EF $\alpha$-NT3, the concentration of NT-3 was $88.80 \pm 27.32$ ng of NT-3 per milligram of soluble protein, compared with $163.07 \pm 54.91 \mathrm{ng}$ of NT-3 per milligram of soluble protein at 1 week, indicating that the expression levels had dropped by 3 weeks. 


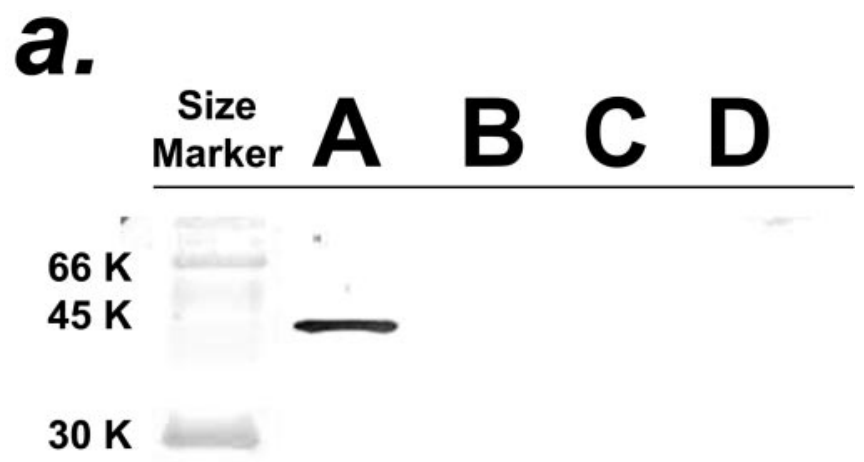

$14.3 \mathrm{~K}$

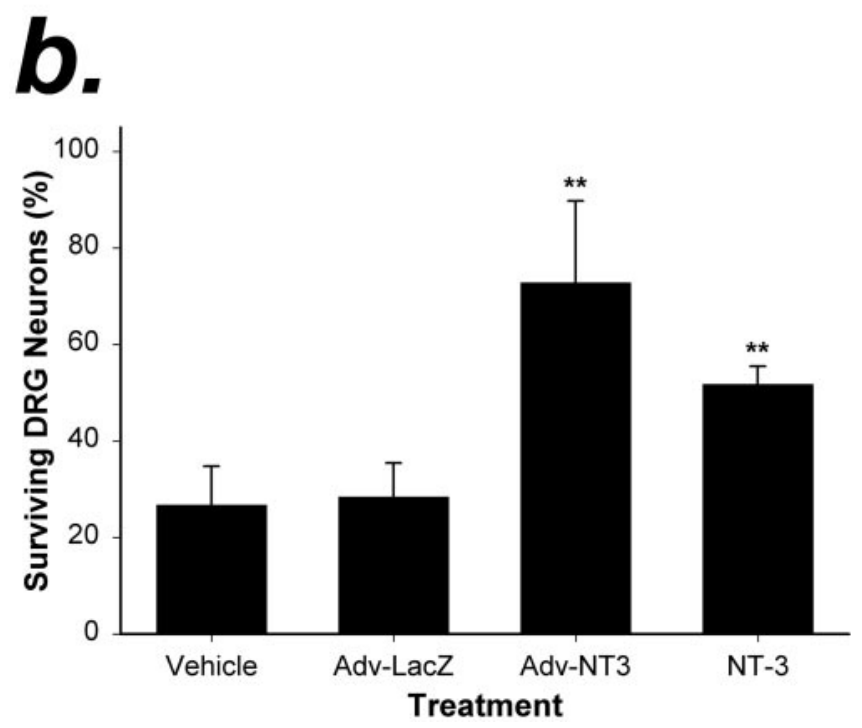

Figure 2. Analysis of NT-3 protein produced by cells transduced with Adv.EF $\alpha$-NT3 in vitro. $a$, Western blot analysis of the conditioned medium of HeLa cells transduced with Adv. HeLa cells were transduced with Adv.EF $\alpha$-NT3 or Adv.EF $\alpha$-LacZ at an MOI of 100. After 12 hr, the medium was replaced with serum-free medium. After $48 \mathrm{hr}$, the conditioned medium was collected. Western blot analysis using an anti-FLAG antibody was performed to detect NT-3FLAG in the medium. Lane A, BAP-FLAG; Iane B, medium from untransduced HeLa cells; Iane C, medium from HeLa cells transduced with Adv.EF $\alpha$-LacZ; lane D, medium from HeLa cells transduced with Adv.EF $\alpha$-NT3. A prominent band that cross-reacted with the anti-FLAG antibody is visible in lane D corresponding to the predicted size of the NT-3-FLAG hybrid protein. $b$, Conditioned medium of HeLa cells transduced with Adv.EF $\alpha$-NT3 supported the survival of DRG neurons. Primary cultures of DRG neurons were cultured in test media for $48 \mathrm{hr}$, and the number of surviving neurons was counted in 10 fields. The test media were as follows: Vehicle, $25 \%$ conditioned medium from cultures of untransduced HeLa cells; Adv-LacZ, 25\% conditioned medium from cultures of Adv.EF $\alpha$-LacZ-transduced HeLa cells; Adv-NT3, 25\% conditioned medium from cultures of Adv.EF $\alpha$-NT3-transduced HeLa cells; and NT-3, $100 \mathrm{ng}$ of NT-3 protein per milliliter of culture medium. The values are means \pm SD of three wells; ${ }^{* *} p<0.01$ (ANOVA followed by the Student-Newman-Keuls test).

To determine whether the retrograde delivery of Adv affected motoneuron survival, we counted the number of motoneurons present in sections of lumbar spinal cord 5 weeks after CSTL and 3 weeks after the sciatic nerve was cut and the Adv delivered. Large, multipolar cells located at the ventral horn of the spinal cord were identified as motoneurons. Comparison of the numbers of motoneurons in the lumbar region sections taken from the lesioned versus the unlesioned side of the spinal cord did not

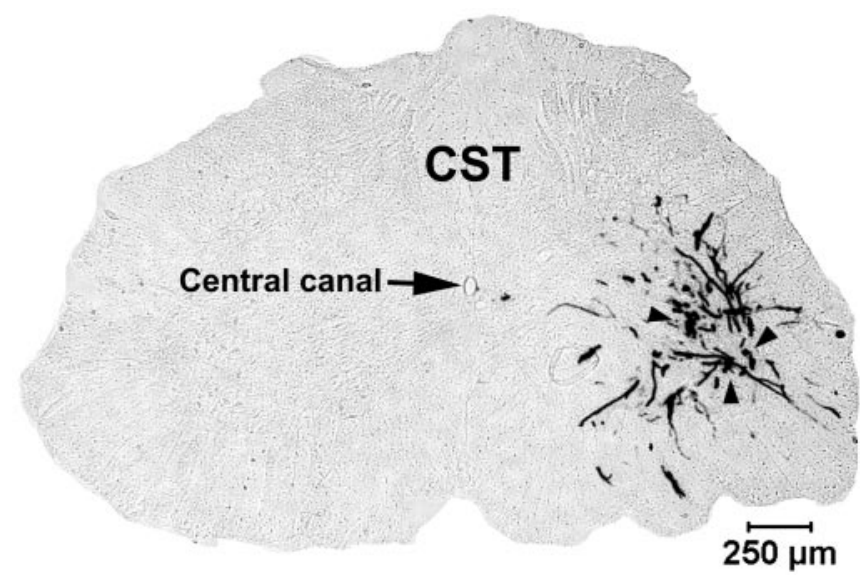

Figure 3. $\quad \beta$-gal is expressed in motoneurons of the lumbar spinal cord. The sciatic nerve was cut, and the proximal stump was inserted into a small chamber filled with Adv.EF $\alpha$-LacZ $(1 \times$ $10^{9}$ infectious units). After $7 \mathrm{~d}$, rats were perfusion-fixed with $4 \% \mathrm{PFA}$, cross sections were cut, and $\beta$-gal was identified by histochemical staining with X-gal. $\beta$-gal-positive neuronal processes are visible extending from transduced motoneurons (arrowheads) to regions close to the midline and central canal.

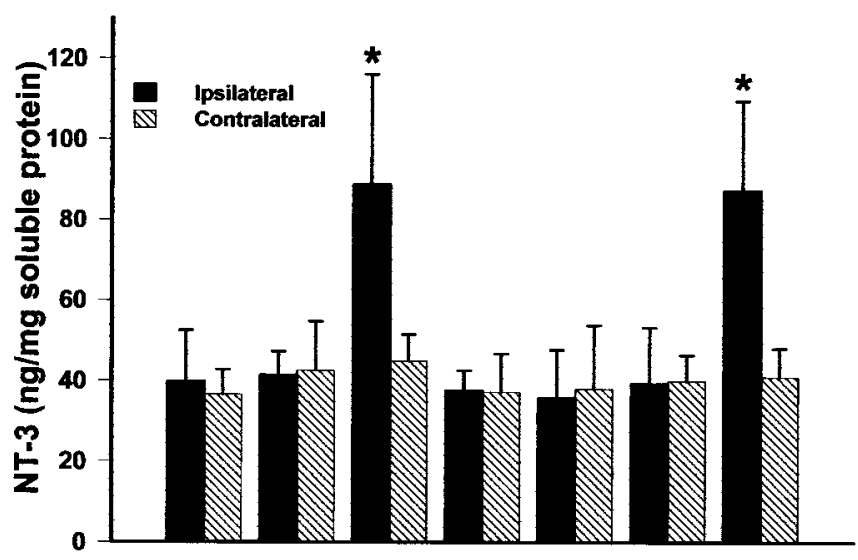

Figure 4. Expression of NT-3 in the L3-L6 lumbar spinal cord 3 weeks after the retrograde delivery of Adv. The concentration of NT-3 in the spinal cord was determined by ELISA (Promega). Values are means $\pm S D ;{ }^{*} p<0.05 ; n=4$ per group (ANOVA followed by the StudentNewman-Keuls test).

show a statistically significant difference ( $p=0.596$; Student's $t$ test; $\mathrm{df}=16 ; t=0.541)$. Nor was there a significant difference $(p=0.989$; ANOVA; $\mathrm{df}=2 ; F=0.0115)$ among the numbers of motoneurons present on the lesioned side in groups treated with Adv.EF $\alpha$-NT3 $[41.33 \pm 4.16$ (mean \pm SD)], Adv.EF $\alpha$-LacZ $(55.00 \pm 7.21)$, PBS $(46.00 \pm 1.00)$, or sham operation $(39.33 \pm$ 13.65). These data, based on measurements in five sections per rat (three rats per group), demonstrate that sciatic nerve sectioning with or without Adv delivery did not influence the survival of motoneurons in lumbar spinal cord within 3 weeks. Immunohistological analysis of the lumbar spinal cord of animals with CSTL and transduced with Adv revealed that ED1-positive macrophages were present in the lesioned CST commensurate with wallerian degeneration of the axotomized axons, but none were present in the unlesioned CST or gray matter (data not shown). This confirms that CSTL at the level of the pyramids and retrograde delivery of Adv through the sciatic nerve did not cause widespread chronic inflammation in the lumbar spinal cord. 

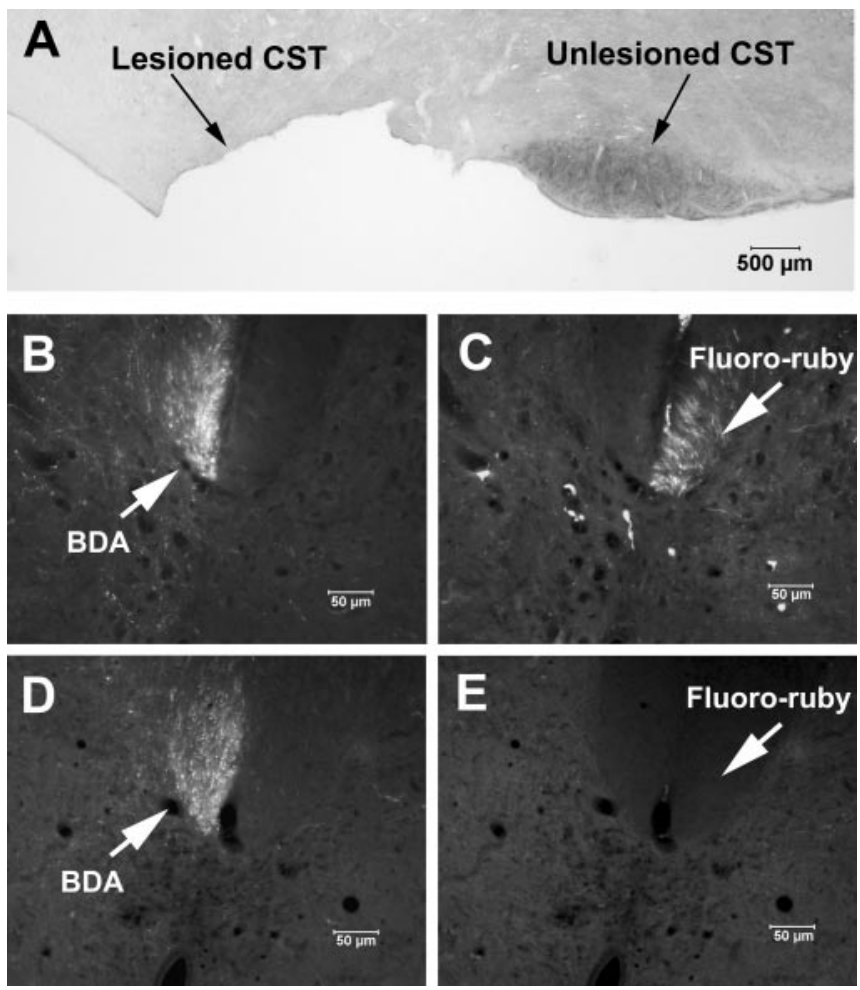

Figure 5. A, Photomicrograph at the level of the lesion site in the pyramids showing the extent of the lesioned CST. The nonlesioned CST is demarcated by the anterograde marker BDA stained with $A B C$ reagent (Vector Laboratories) and DAB. $B-E$, Completeness of the CST lesion demonstrated by anterograde markers in the lumbar spinal cord of unlesioned $(B, C)$ and lesioned $(D, E)$ animals. The unlesioned CST was traced with BDA and visualized with Alexa Fluor 488-conjugated streptavidin; the lesioned CST was traced with Fluoro-ruby. B, Section from a normal rat (sham surgery) showing the unlesioned CST labeled with BDA.C, The same section as $B$, showing the CST positive for Fluoro-ruby. D, Section from an animal with a complete CSTL, showing the unlesioned side of CST positive for BDA. E, Same section as D showing absence of Fluoro-ruby in the lesioned CST, indicating that the CSTL was complete.

\section{Completeness of CST lesion}

As shown in Figure $5 A$, the left CST was completely removed at the pyramidal lesion site. The completeness of the CSTL was further examined in sections of lumbar spinal cord. In animals without CSTL or with incomplete CSTL, Fluoro-ruby was visible in the right CST in the lumbar spinal cord, as shown in Figure $5 C$. For animals with complete CSTL, Fluoro-ruby was not present in the right CST (Fig. 5E). BDA efficiently labeled the unlesioned CST (Fig. 5B,D).

\section{Sprouting of contralateral CST axons after in situ expression} of NT-3

In the rat, most CST fibers cross the midline at the pyramidal decussation within the brainstem and project into the contralateral dorsal funiculus of the spinal cord (Fig. 1); however, a small number of fibers do not cross at the pyramidal decussation and project ipsilaterally into the ventral and dorsal funiculi (Brosamle and Schwab, 1997; Z'Graggen et al., 1998). We tested whether sustained in situ expression of NT-3 in the spinal cord ipsilateral to the lesioned CST would elicit axonal sprouting from the intact contralateral CST. Seven groups of rats that received either CSTL and Adv.EF $\alpha$-NT3 (CSTL plus NT-3; $n=8$ ), CSTL and Adv.EF $\alpha$-LacZ (CSTL plus LacZ; $n=8$ ), CSTL and vehicle (CSTL plus PBS; $n=4$ ), no CSTL and Adv.EF $\alpha$-NT3 (sham plus NT-3; $n=5$ ), no CSTL and Adv.EF $\alpha$-LacZ (sham plus LacZ; $n=5$ ), no CSTL and PBS (sham plus PBS; $n=2$ ), or no CSTL or sciatic
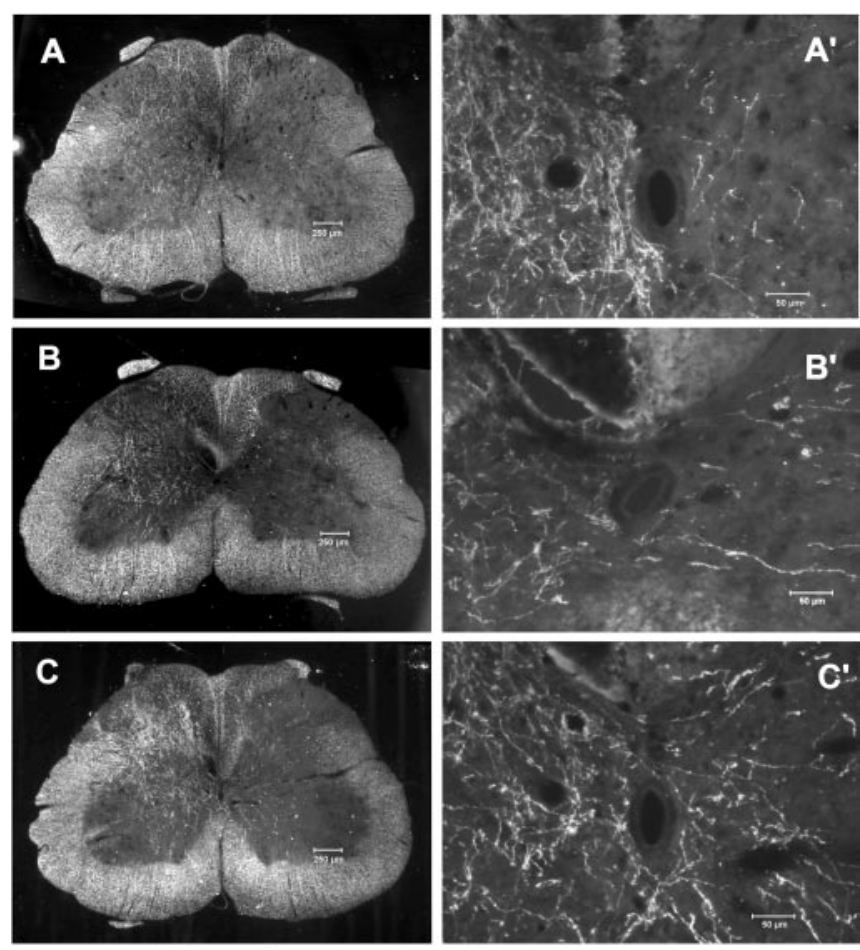

Figure 6. Sprouting of CST axons across the midline in the spinal cord after CSTL and Adv.EF $\alpha$-NT3 transduction of motoneurons. Rats with unilateral CSTL were treated with Adv.EF $\alpha$-NT3 or Adv.EF $\alpha$-LacZ, whereas the unlesioned CST was labeled with BDA. Dark-field photomicrographs of spinal cord cross sections showed the unlesioned CST axons. A, Section from a normal rat (sham surgery). B, Section from an Adv.EF $\alpha$-LacZ-treated rat. C, Section from an $\mathrm{Adv}$.EF $\alpha$-NT3-treated rat. $A^{\prime}-C^{\prime}$, Higher-power photomicrographs of the regions around the central canal. C, BDA-labeled CST neurites can be seen arising from the intact CST, traversing the midline, and growing into the gray matter of the lesioned side of the spinal cord.

nerve cut (normal; $n=3$ ) were sampled. Under dark-field illumination, cross sections taken from the region of the lumbar spinal cord showed few BDA-labeled CST axons projecting from the intact CST to the lesioned side of the spinal cord in either the normal (Fig. $6 A, A^{\prime}$ ) or CSTL plus LacZ groups (Fig. $6 B, B^{\prime}$ ). In contrast, considerably more axons projected across the midline into the lesioned side of the spinal cord in the CSTL plus NT-3 group (Fig. 6C, $C^{\prime}$ ).

\section{Quantification of sprouting of contralateral CST axons after in situ expression of NT-3}

The number of CST axons that projected across the midline from the unlesioned CST was measured in sections taken from the seven experimental groups listed above. It is likely that the efficiency of anterograde labeling of CST axons with BDA varied among animals, which in turn would result in a variable amount of axons visible in spinal cord sections. Thus, simply counting the axons that passed through the midline would not represent the actual degree of sprouting in the experimental groups. To compensate for variable labeling, we counted the number of BDAlabeled axons in two regions of the unlesioned side $\left(N_{1}+N_{2}\right)$ as well as the number of axons crossing the midline $(M)$ in sections taken from the lumbar region of the spinal cord (see Materials and Methods) (Fig. 7a). Because the value for $N_{1}+N_{2}$ represented the degree of BDA labeling for each animal, $M / N_{1}+N_{2}$ represented the number of axons crossing the midline relative to the BDA labeling. Axons lateral to the midline on the lesioned side $\left(L_{1}\right.$ and $\left.L_{2}\right)$ (Fig. $7 a$ ) were also counted, so that the ratios of $L_{1} / N_{1}+N_{2}$ and $L_{2} / N_{1}+N_{2}$ represent the axons that crossed the 


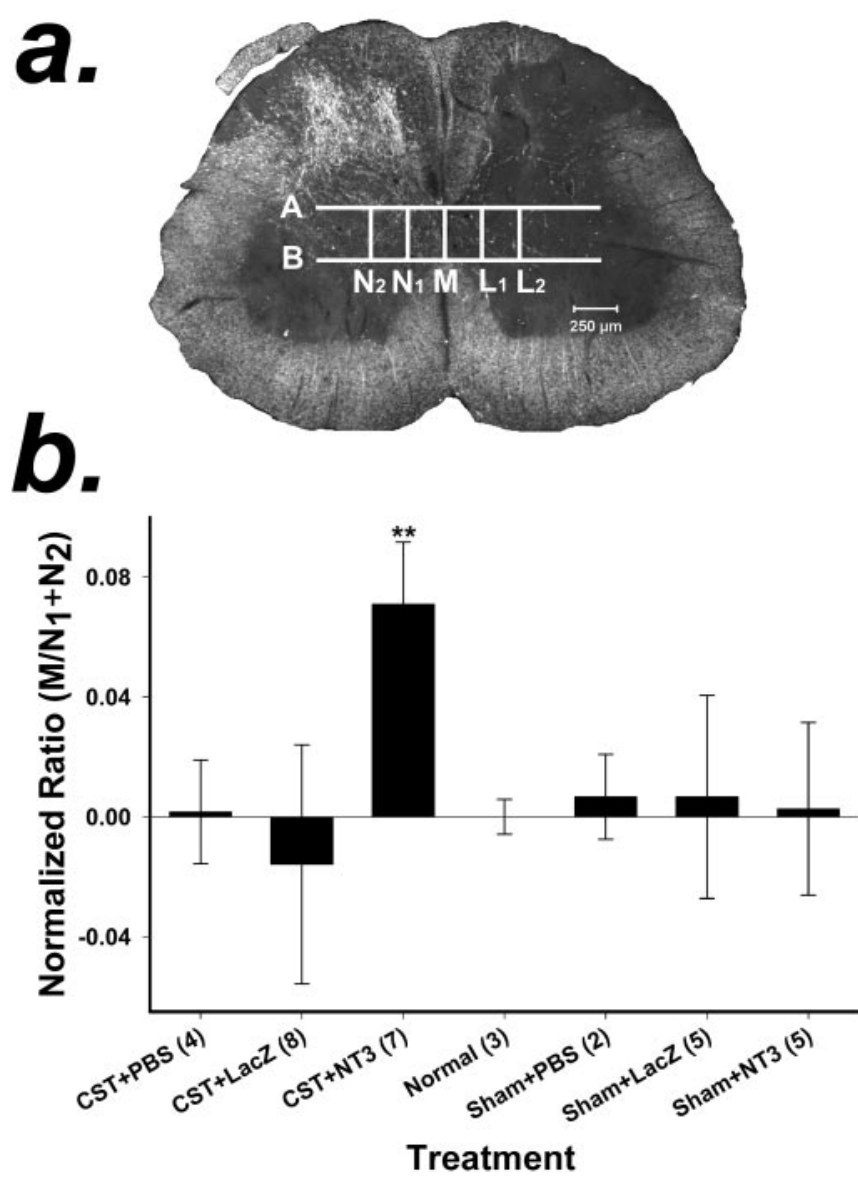

Figure 7. a, Method for quantification of axonal sprouting from the CST across the midline in the spinal cord. Dark-field photomicrographs were taken of each spinal cord section. Five vertical and two horizontal lines were drawn on each photomicrograph of a spinal cord section as reference points for counting axons. $M$ was drawn through the midline, $N_{1}$ was drawn just lateral to the funiculus of the CST and parallel to $M$, and $N_{2}$ was drawn parallel to $M$ and two times the distance between $N_{1}$ and $M . L_{1}$ and $L_{2}$ were drawn on the lesioned side of the spinal cord and corresponded to lines $N_{1}$ and $N_{2}$. Lines $A$ and $B$ were drawn perpendicular to the dorsoventral axis of the cord. $A$ was drawn just below the dorsal columns, and $B$ was drawn just above the ventral columns. Axons that crossed $M, N_{1}, N_{2}, L_{1}$, and $L_{2}$ within the boundaries of $A$ and $B$ were counted. $b$, Quantification of CST axons crossing the midline in response to the local expression of NT-3. BDA-positive axons were counted at the midline $(M)$ and at two sites $\left(N_{1}\right.$ and $N_{2}$ ) in the lateral gray matter of the spinal cord on the side of the unlesioned CST (see Materials and Methods). The ratio of the axons that crossed the midline $(M)$ to those that crossed the two sites in the lateral gray matter $\left(N_{1}+N_{2}\right)$ was computed $\left(M / N_{1}+N_{2}\right)$ to compensate for any variation in the degree of anterograde labeling of the CST. The ratios of the treatment groups were normalized to the ratio of the normal animal group, which was set to zero. A positive value indicates that more axons crossed the midline compared with normal, unlesioned animals; a negative value indicates that fewer axons crossed the midline compared with normal animals. Values are means \pm SD; ${ }^{* *} p<0.01$ (ANOVA followed by the StudentNewman-Keuls test). Numbers in parentheses represent the number of animals per group.

midline and reached the lines of $L_{1}$ and $L_{2}$ relative to the degree of BDA labeling. There was no difference in the $M / N_{1}+N_{2}$ ratio among groups that did not receive a CSTL lesion (ANOVA followed by the Student-Newman-Keuls test; $p<0.01 ; F=6.313$; $\mathrm{df}=6$; $\left.\operatorname{power}_{(\alpha=0.05)}=0.984\right)($ Fig. $7 b)$, demonstrating that in the absence of denervation, in situ expression of NT-3 had no effect on axonal sprouting. The CSTL plus NT-3 group had a significantly greater $M / N_{1}+N_{2}$ ratio compared with all other groups (ANOVA followed by the Student-Newman-Keuls test; $p<0.01 ; F=6.313$; df $=6$ ). There was a slight, statistically insignificant reduction in the number of axons that crossed the midline in the CSTL plus LacZ animals. It is not clear whether this represents a true treatment effect within the spinal cord or in the periphery or whether it is attributable to the variation in animals. The $L_{1} / N_{1}+N_{2}$ and $L_{2} / N_{1}+N_{2}$ ratios in the CSTL plus NT-3 group were not statistically different from those of the CSTL plus LacZ and CSTL plus PBS groups (data not shown). These findings demonstrate that local expression of NT-3 will elicit and support sprouting of CST axons into a region of traumatically denervated spinal cord, but not when both CSTs are intact.

\section{Discussion}

\section{Adenovirus-mediated expression of biologically active NT-3 in vitro}

Conditioned medium from HeLa cells transduced with Adv.EF $\alpha$ NT3 at an MOI of 100 sustained the survival of DRGs in vitro, demonstrating that the vector directs the expression and release of biologically active NT-3. HeLa cells transduced with Adv.EF $\alpha$ NT3 at an MOI of 100 produced and secreted into the medium $760 \mathrm{ng}$ of NT-3 per $10^{6}$ cells per day, which is $>10$-fold higher than the average rate of $43 \mathrm{ng}$ of neurotrophic factor per $10^{6}$ cells per day that we measured in Adv constructs in which neurotrophic factor genes were driven by the RSV promoter (Baumgartner and Shine, 1997). This higher expression rate of biologically active NT-3 coupled with the longer duration of expression reported by others (Guo et al., 1996) provided the rationale for locally expressing NT-3 in the spinal cord of rats with the EF $\alpha$ promoter.

\section{Retrograde transport of Adv.EF $\alpha$-NT3 and expression of NT-3 in the spinal cord}

ELISA analyses of spinal cord tissue showed a threefold greater concentration of NT-3 at 1 week and a twofold greater concentration at 3 weeks on the side of the spinal cord on which motoneurons had been transduced with Adv.EF $\alpha$-NT3 than on the untreated contralateral side or when given control treatments. This finding, coupled with the robust expression of LacZ in motoneurons transduced with Adv.EF $\alpha-L a c Z$ and increased contralateral sprouting from the intact CST, supports the conclusion that biologically active NT-3 is expressed and released by transduced motoneurons in the spinal cord. However, as in previous experiments with Adv.RSV-nf (Baumgartner and Shine, 1997), we were unable to detect the expression of NT-3 or the FLAG marker sequence by standard immunohistochemical techniques. The inability to detect NT-3 in the spinal cord may have resulted from low expression rates, short half-life, low concentrations attributable to rapid release and diffusion of the molecule, or posttranslational modifications that masked the antigenic sites and typify the general difficulty in localizing physiological levels of neurotrophic factors in neural tissue by immunocytochemistry (Zhou et al., 1994).

To avoid vector-associated inflammation, we exploited the fact that Adv is transported from the periphery to the CNS by retrograde transport (Ridoux et al., 1994; Baumgartner and Shine, 1997; Garcia-Valenzuela et al., 1997; Turner et al., 2001). Because the viral capsid proteins that would elicit an immune response (Thomas et al., 2001) are concealed within the axon and neuronal cell body, they escape immune surveillance. This concealment and the use of rat NT-3 cDNA eliminated the possibility that delivery of Adv-EF $\alpha$-NT3 to the spinal cord would induce a severe immune response, although we did observe inflammation in the periphery of the delivery site (data not shown). In addition, we did not observe any neuronal death associated with Adv transduction for up to 3 weeks after delivery of the vectors.

In our previous research, we targeted neurotrophic factors to 
the motoneurons of neonatal rats by injecting them with Adv.RSV-nf directly into muscle, where the vectors were efficiently taken up by peripheral nerves and transported to motoneurons (Baumgartner and Shine, 1997). This route of delivery was less efficient in adult rats compared with neonatal rats, so that few motoneurons were transduced with Adv when injected into muscle (data not shown). We surmise that an impediment to efficient motoneuron targeting in adult animals is the increased myelination or ensheathment of axons by Schwann cells and the increased stroma surrounding synaptic clefts, which would block the access of Adv to axons. To overcome this obstacle, we placed the proximal stump of the cut sciatic nerve in a capsule containing Adv.EF $\alpha$-NT3, so that a sufficient quantity of the vector was exposed to bare axons. Although solving the problem of inefficient transduction in adult rats, this strategy precluded subsequent functional analyses.

Histochemical staining for $\beta$-gal activity in motoneurons transduced with Adv.EF $\alpha$-LacZ confirmed reports by us and others that Advs are taken up by peripheral axons and transported in a retrograde manner to motoneurons, in which they direct transgene expression (Ridoux et al., 1994; Baumgartner and Shine, 1997; Garcia-Valenzuela et al., 1997; Turner et al., 2001). Histochemical staining for $\beta$-gal also revealed that the transduced motoneurons had dendrites extending close to the midline of the spinal cord. This observation suggests that if transduced motoneurons are capable of releasing NT-3 from their dendrites, then the distance that the factor must diffuse to affect CST axons would be as short as $100 \mu \mathrm{m}$. It is likely that DRG neurons were transduced by the Adv, so that central projecting axons may release NT-3 in the dorsal columns. Because the motoneuron pool is localized in a discrete area of the ventral horn, there would likely be a concentration gradient that increases as the distance to the motoneuron pool decreases, thus acting to direct sprouting CST axons into the ventral horn.

\section{Sprouting from the intact CST is induced by local expression of NT-3}

The principal observation of our experiments is that the local expression of NT-3 by motoneurons induced and supported the sprouting of axons from the contralateral CST. It is notable that the sprouting was (1) observed, suggesting that overexpression of NT-3 induced the axons to overcome or evade any negative factors that may inhibit axonal growth; (2) induced in uninjured axons, demonstrating that these observations represent neurotrophic factor-induced plasticity rather than regeneration; and (3) present only when the ipsilateral CST was lesioned, suggesting that other factors play a role in the observed plasticity.

Lesioning the CST at the level of the pyramids avoided conditions associated with other spinal cord lesion models, such as inflammation, secondary cell death, vascular disruption, and connective-tissue disruption, which could confound analysis of the effect of NT-3 on axonal plasticity. Because the lesion was distal to the region of analysis, no trauma-associated inhibitory molecules such as Nogo or myelin-associated glycoprotein were released or upregulated (such as chondroitin sulfate proteoglycans) that could have blocked axonal projection from the CST (Schwab, 2002). In the absence of a trauma-induced inhibitory environment, axons grew through normal gray matter. Axonal plasticity in the undamaged spinal cord has been reported in other experimental contexts. Presenting a blocking antibody (IN-1) to the inhibitory molecule Nogo elicited axonal growth from the intact CST in the CSTL model (Thallmair et al., 1998). Interestingly, the inhibition of Nogo with IN-1 had the same effect on axonal plasticity in the model as the local expression of NT-3, suggesting that more than one factor plays a role in inhibiting axonal plasticity, and that modification of one is sufficient to release the process. Infusing inosine supported robust contralateral sprouting of intact CST axons into the white matter in the region of the lesioned CST in the CSTL model (Benowitz et al., 1999). The fact that the inosine-induced axonal plasticity passed from white matter tract to white matter tract, in contrast to our observation that axons preferentially grew in the gray matter, suggests that the two treatments target different pathways or mechanisms. DRG neurons carefully grafted into the spinal cord white matter grew up to $11 \mathrm{~mm}$ within $10 \mathrm{~d}$, but only when the host tissue was minimally disturbed and no scar tissue had formed (Davies et al., 1999). It remains to be seen whether local expression of NT-3 would coax axons to cross an area of damaged tissue that would contain higher concentrations of inhibitory molecules. These data cannot address whether the newly sprouted CST axons had formed functional synapses with the motoneurons, because cutting the sciatic nerve precluded behavioral testing. However, other laboratories have reported that contralateral CST sprouting will result in the restoration of function (Thallmair et al., 1998; von Meyenburg et al., 1998; Z'Graggen et al., 1998); hence, functional synapses may have formed in the animals in our experiments.

That we observed axonal plasticity only when the ipsilateral motoneurons were denervated by lesioning the ipsilateral CST suggests that NT-3 alone is insufficient to elicit and support this axonal plasticity. Additional factors present in the denervated side of the spinal cord may signal the intact CST to either sprout collaterals or grow toward the denervated side and that NT-3 acts in concert with these signals to establish the contralateral axonal projections. Because we measured the degree of sprouting at only one time point ( 5 weeks after CSTL and 3 weeks after the beginning of NT-3 overexpression), it is not clear whether the number of axons crossing the midline is stable or are increasing or decreasing. Nor is it known whether CST axons would respond differently if NT-3 were expressed at other times after CSTL.

\section{Conclusions}

These data demonstrate that local, sustained expression of NT-3 by motoneurons will support sprouting of intact CST axons after they have lost their normal CST innervation. In addition, they suggest that CNS neurons have the capacity to respond to trauma-induced denervation through axonal plasticity. Hence, it is possible that interventions based on gene delivery would promote limited functional recovery from neural trauma by eliciting plasticity changes in intact nervous system tissue in addition to regenerating damaged tissue.

\section{References}

Baumgartner BJ, Shine HD (1997) Targeted transduction of CNS neurons with adenoviral vectors carrying neurotrophic genes confers neuroprotection that exceeds the transduced population. J Neurosci 17:6504-6511.

Baumgartner BJ, Shine HD (1998a) Neuroprotection of spinal motoneurons following targeted transduction with an adenoviral vector carrying the gene for glial cell-line derived neurotrophic factor. Exp Neurol 153:102-112.

Baumgartner BJ, Shine HD (1998b) Permanent rescue of lesioned neonatal motoneurons and enhanced axonal regeneration by adenovirusmediated expression of glial cell-line-derived neurotrophic factor. J Neurosci Res 54:766-777.

Benowitz LI, Goldberg DE, Madsen JR, Soni D, Irwin N (1999) Inosine stimulates extensive axon collateral growth in the rat corticospinal tract after injury. Proc Natl Acad Sci USA 96:13486-13490. 
Brösamle C, Schwab ME (1997) Cells of origin, course, and termination patterns of the ventral, uncrossed component of the mature rat corticospinal tract. J Comp Neurol 386:293-303.

Davies AM (1989) Neurotrophic factor bioassay using dissociated neurons. In: Nerve growth factors (Rush RA, ed), pp 95-109. New York: Wiley.

Davies SJA, Goucher D, Doller C, Silver J (1999) Robust regeneration of adult sensory axons in degenerating white matter of the adult rat spinal cord. J Neurosci 19:5810-5822.

Garcia-Valenzuela E, Rayanade R, Perales JC, Davidson CA, Hanson RW, Sharma SC (1997) Axon-mediated gene transfer of retinal ganglion cell in vivo. J Neurobiol 32:111-122.

Graham F, Prevec L (1991) Manipulation of adenovirus vectors. In: Methods in molecular biology: gene transfer and expression protocols (Murray EJ, ed), pp 109-128. Clifton, NJ: Humana.

Grill RJ, Blesch A, Tuszynski MH (1997) Robust growth of chronically injured spinal cord axons induced by grafts of genetically modified NGFsecreting cells. Exp Neurol 148:444-452.

Guo ZS, Wang L-H, Eisensmith RC, Woo SLC (1996) Evaluation of promoter strength for hepatic gene expression in vivo following adenovirusmediated gene transfer. Gene Ther 3:802-810.

Hopp T, Prickett K, Price C, Libby R, March C, Cerretti P, Urdal D, Conlon P (1988) A short marker sequence useful for recombinant protein identification and purification. Biotech 6:1205-1210.

Kim DW, Uetsuki T, Kaziro Y, Yamaguchi N, Sugano S (1990) Use of the human elongation factor $1 \alpha$ promoter as a versatile and efficient expression system. Gene 91:217-223.

Kim DW, Harada T, Saito I, Miyamura T (1993) An efficient expression vector for stable expression in human liver cells. Gene 134:307-308.

Ridoux V, Robert JJ, Zhang X, Perricaudet M, Mallet J, Le Gal La Salle G
(1994) Adenoviral vectors as functional retrograde neuronal tracers. Brain Res 648:171-175.

Schnell L, Schneider R, Kolbeck R, Barde Y-A, Schwab ME (1994) Neurotrophin-3 enhances sprouting of corticospinal tract during development and after adult spinal cord lesion. Nature 367:170-173.

Schwab ME (2002) Repairing the injured spinal cord. Science 295:1029-1031.

Thallmair M, Metz GAS, Z'Graggen WJ, Raineteau O, Kartje GL, Schwab ME (1998) Neurite growth inhibitors restrict plasticity and functional recovery following corticospinal tract lesions. Nat Neurosci 1:124-131.

Thomas CE, Birkett D, Anozie I, Castro MG, Lowenstein PR (2001) Acute direct adenoviral vector cytotoxicity and chronic, but not acute, inflammatory responses correlate with decreased vector-mediated transgene expression in the brain. Mol Ther 3:36-46.

Turner DE, Noordmans AJ, Feldman EL, Boulis NM (2001) Remote adenoviral gene delivery to the spinal cord: contralateral delivery and reinjection. Neurosurgery 48:1309-1316.

Vanek P, Thallmair M, Schwab ME, Kapfhammer JP (1998) Increased lesion-induced sprouting of corticospinal fibers in the myelin-free rat spinal cord. Eur J Neurosci 10:45-56.

von Meyenburg J, Brösamle C, Metz GA, Schwab ME (1998) Regeneration and sprouting of chronically injured corticospinal tract fibers in adult rats promoted by NT-3 and the mAb IN-1, which neutralizes myelinassociated neurite growth inhibitors. Exp Neurol 154:583-594.

Z'Graggen WJ, Metz GA, Kartje GL, Thallmair M, Schwab ME (1998) Functional recovery and enhanced corticofugal plasticity after unilateral pyramidal tract lesion and blockade of myelin-associated neurite growth inhibitors in adult rats. J Neurosci 18:4744-4757.

Zhou XF, Zettler C, Rush RA (1994) An improved procedure for the immunohistochemical localization of nerve growth factor-like immunoreactivity. J Neurosci Methods 54:95-102. 